Comm. in Asteroseismology

Vol. 153, 2008

\title{
MOST found evidence for solar-type oscillations in the K2 giant star HD 20884
}

\author{
T. Kallinger ${ }^{1}$, D. B. Guenther ${ }^{2}$, W. W. Weiss ${ }^{1}$, M. Hareter ${ }^{1}$, \\ J. M. Matthews ${ }^{3}$, R. Kuschnig ${ }^{1}$, P. Reegen ${ }^{1}$, G. A. H. Walker ${ }^{3}$, \\ S. M. Rucinski ${ }^{4}$, A. F. J. Moffat ${ }^{5}$, and D. Sasselov 6 \\ ${ }^{1}$ Institut für Astronomie, Universität Wien \\ Türkenschanzstrasse 17, 1180 Vienna, Austria \\ 2 Institute for Computational Astrophysics, \\ Department of Astronomy and Physics, \\ Saint Marys University, Halifax, NS B3H 3C3, Canada \\ 3 Department of Physics and Astronomy, \\ University of British Columbia, 6224 Agricultural Road, \\ Vancouver, BC V6T 1Z1, Canada \\ ${ }^{4}$ Department of Astronomy and Physics, \\ David Dunlap Observatory, University of Toronto, \\ P. O. Box 360, Richmond Hill, ON L4C 4Y6, Canada \\ ${ }^{5}$ Départment de Physique, Université de Montréal \\ C. P. 6128, Succursale : Centre-Ville, Montréal, QC H3C 3J7, \\ Obs. Astro. du Mont Mégantic, Canada \\ ${ }^{6}$ Harvard-Smithsonian Center for Astrophysics, \\ 60 Garden Street, Cambridge, MA 02138
}

\section{Comments by the editor:}

The main message of the following paper is to report the detection of nonradial pulsation in a $\mathrm{K}$ giant. This is an important and controversial topic.

A key issue for this investigation is the reliability of the extracted frequencies. Your editor has spent his life with Fourier amplitude spectra of high-precision ground-based photometry. From that experience, Figure 3 of the paper represents the natural result of instrumental instabilities and atmospheric transparency changes and does not deserve a second look. However, these are satellite observations without the typical ground-based difficulties - but there may be different problems.

The statistical analyses can only help us in a limited way. It does not matter much whether one applies my favorite criterion of amplitude signal/noise ratio $\geq 4$, the SigSpec values, or another method. All these statistical analyses rely on assumptions of the nature of noise present in the data. Again, in ground-based photometry, we have found that the statistics are not reliable for low-frequency peaks. The obvious method to verify that certain peaks are not noise is to look at a comparison sample: the authors provide the results for another star with a simpler light curve. In my opinion, the issue is not yet solved. 


\begin{abstract}
We found evidence for radial p-modes and nonradial mixed modes in the oscillation spectrum of the K giant HD 20884 based on 20.6 days of nearly continuous high-precision photometry obtained by the Canadian microsatellite MOST ${ }^{1}$. Oscillation frequencies range from $5-31 \mu \mathrm{Hz}$ (periods of about $2.3 \mathrm{~d}-9 \mathrm{hr}$ ) with luminosity amplitudes between about 300 and 950 ppm and mode lifetimes exceeding 10 days are indicated. The mode identifications are based on searches of a large grid of models for a best fit to the frequencies and temperature of HD 20884. The latter is better constrained now by spectroscopy obtained at the David Dunlap Observatory as part of this work.
\end{abstract}

Individual Objects: HD 20884, HD 20790, $\kappa^{1}$ Ceti, $\epsilon$ Oph, $\xi$ Hya, HD 146490

\title{
Introduction
}

Our understanding of the Sun's structure has been revolutionised over the last three decades by helioseismology. This technique allows investigations of the solar interior by observing $p$-modes at the stellar surface. Observing sun-like oscillations in other stars was hampered for a long time by the extremely small pulsation amplitudes. For stars cooler and more luminous than the Sun, the expected amplitudes are greater and should be more easily observable. However, the larger radii of red giants extend their pulsation periods from $\sim 5$ minutes in the Sun to a range of several hours to several days. This again complicates groundbased detections and frequency identifications, especially due to daily aliasing.

As ultra-precise rapid photometry from space and high-precision radial velocity measurements from the ground became available, detections of stochastically driven oscillations were reported for several giants (see Bouchy \& Carrier 2003 for a review). Examples include the G9.5 giant $\epsilon$ Oph, based on radial velocities from the CORALIE and ELODIE spectrographs (De Ridder et al. 2006) and Fabry Imaging photometry from the MOST satellite (Barban et al. 2007), and the $\mathrm{G} 7$ giant $\xi \mathrm{Hya}$, based on radial velocity measurements with the CORALIE spectrograph (Frandsen et al. 2002), or the K2.5 giant star GSC 09137-03505 where Kallinger et al. (2005) found oscillations, based on Fine Guidance Sensor photometry by the Hubble Space Telescope.

If pulsating red giants do indeed have radial and observable nonradial modes with relatively long lifetimes, then these modes will enable to constrain the deep interior of red giant stars, as well as set limits to the excitation mechanisms. But recent investigations (e.g., Barban et al. 2007 or Stello et al. 2006 ) suggest that only radial modes with very short lifetimes ( $<3$ days) should be observable in red giants. On the other hand, Kallinger et al. (2008b) report strong evidence for the existence of radial and nonradial modes in $\epsilon$ Oph (with lifetimes longer than 10 days) based on

\footnotetext{
${ }^{1}$ Based on data from the MOST (Microvariability \& Oscillation of STars) satellite, a Canadian Space Agency mission jointly operated by Dynacon, Inc., the University of Toronto Institute of Aerospace Studies, and the University of British Columbia, with assistance from the University of Vienna, Austria.
} 
pulsation model fits to the re-examined MOST photometry. This is also supported by Hekker et al. (2006) who found line profile variations suggesting nonradial modes.

However, the theoretical situation is unclear. Dziembowski et al. (2001) show the existence of strongly trapped unstable nonradial mixed $g$-modes $/ p$-modes between very closely spaced modes of the same degree $l$ following the same pattern of frequency spacing as radial modes. Only in their linear stability analysis, including the effects of turbulent pressure based on the mixing-length formalism, do they predict amplitudes of radial modes for the sub-giant $\alpha \mathrm{UMa}$ to be much smaller than observed, with the nonradial modes predicted to be even smaller.

In this paper, we present evidence for damped and stochastically excited radial and nonradial oscillations in the K giant star HD 20884, based on MOST Guide Star photometry obtained as part of the 2004 campaign on the active solar-type star $\kappa^{1}$ Ceti (see Rucinski et al. 2004 for the results of the 2003 campaign). Linear adiabatic radial and nonradial eigenfrequencies were computed for a large grid of stellar models with standard parameters. 14 of the 17 most significant frequencies are identifiable as low-degree $(l=0,1$ and 2$) p$-modes and mixed modes. The resulting best fit for a $\mathrm{K}$ giant stellar model is compatible with spectroscopic constraints based on David Dunlap Observatory spectra obtained for this work. Furthermore, we find strong evidence that the mode lifetimes exceed about 10 days, which is consistent with theoretical predictions for a K giant like HD 20884.

\section{Observations}

MOST (launched on June 30, 2003) contains a CCD photometer fed by a $15 \mathrm{~cm}$ Rumak-Maksutov telescope through a custom broadband optical filter (350 - 750 $\mathrm{nm}$ ). MOST's Sun-synchronous $820 \mathrm{~km}$ polar orbit (period $=101.4 \mathrm{~min}$ ) enables uninterrupted monitoring of a star in its Continuous Viewing Zone $\left(+36^{\circ} \geq \delta \geq-18^{\circ}\right)$ for up to 8 weeks. A pre-launch summary of the mission is given by Walker et al. (2003) and on-orbit science operations are described by Matthews et al. (2004). MOST can obtain three types of photometry for multiple targets simultaneously. Its original primary observing mode is Fabry Imaging, in which the telescope entrance pupil, illuminated by a bright target star, is projected into the Science CCD by a Fabry microlens as an annulus covering about 1300 pixels (Reegen et al. 2006). The second mode is Direct Imaging, in which defocussed images of stars near the primary target are recorded directly on the Science CCD (Rowe et al. 2006). The third mode is Guide Star Photometry, in which intensity measurements are recorded for stars used by MOST's Attitude Control System (ACS) with the Startracker or Science CCD (see Walker et al. 2005 and Aerts et al. 2006).

\section{Guide Star photometry}

In October 2004, MOST reobserved the active G5 dwarf $\kappa^{1}$ Ceti (Walker et al. 2007), monitoring it continuously for 20.6 days to follow its spot activity and the differential rotation measured by MOST in 2003 (Rucinski et al. 2004). Four fainter stars in the field were used as guide stars; they are listed as G0 - G3 in Table 1. The ability to obtain photometry from MOST guide stars was implemented a few months before and had not been possible for the $2003 \kappa^{1}$ Ceti observations. 
Table 1: The guide stars observed during the $\kappa^{1}$ Ceti 2004 campaign. The spectral classifications (based on spectra from this work) are determined to \pm 2 sub-types.

\begin{tabular}{rrrr}
\hline & ID & V & Spectral type \\
\hline G0 & HD 20884 & 7.47 & K2 III \\
G1 & HD 20790 & 8.90 & F4 IV \\
G2 & GSC 00060-00969 & 10.20 & F8 \\
G3 & GSC 00060-00845 & 9.70 & K2 III \\
\hline
\end{tabular}

To point the MOST telescope, its ACS system can determine the relative positions of up to about 20 guide stars on a $1 \mathrm{~K} \times 1 \mathrm{~K} \mathrm{CCD}$, at a sampling rate of about once per second. A window of $20 \times 20$ pixels (about 1 square arcmin) is centred on each guide star. The background intensity is estimated from the first and last row of pixels inside each window and is automatically subtracted onboard - properly weighted - from the total intensity of all the window pixels, resulting in a single guide star intensity.

For the $\kappa^{1}$ Ceti 2004 run, the guide star readings were binned in groups of 29 to give an effective integration time of $26.25 \mathrm{~s}$ and a sampling rate of $35 \mathrm{~s}$. A total of 48,967 binned measurements were obtained for each guide star with an overall duty cycle of about $96 \%$.

MOST has a high pointing stability for a microsat of such low inertia: an average jitter of about 0.6 arcsec for this run, which is about 5 times smaller than the size of an individual pixel. Therefore, pixel-to-pixel sensitivity variations are negligible in the guide star photometry. However, there are variations in the background due to scattered Earthshine which are not entirely removed by the onboard processing. MOST was not originally designed as a differential experiment to explore very long timescales, but thanks to its compact design and stable thermal environment, the instrument's guide star photometry can be sensitive to variations with timescales of weeks and amplitudes of only a millimag or less. At the time of writing, MOST has collected about 560 extensive light curves of about 480 guide stars, providing detailed experience with the quality of the photometry. About $75 \%$ of this MOST guide star sample shows no variability above the point-to-point scatter at timescales shorter than a few days.

Examples of intrinsically variable and constant MOST guide stars at levels of precision presented in this paper can be found in Saio et al. (2006) and Aerts et al. (2006).

\section{The light curve of HD 20884}

None of the guide stars (Table 1 ) were known to be variable prior to the MOST 2004 $\kappa^{1}$ Ceti observations. The two brightest exhibited obvious intrinsic variability in the MOST photometry. The first, HD 20884 (G0), has the most complex variability, with timescales of hours to days. The second, HD 20790 (G1), more than a magnitude fainter than HD 20884 shows the characteristic variation signature of a $\gamma$ Doradus pulsator (see Handler 2005 for a review of these stars) with periods of a few hours. 
The other two stars are even fainter and one (G2) may show evidence of lowfrequency variability, but the Poisson statistics and the low $\mathrm{S} / \mathrm{N}$ relative to the background make the data for G2 and G3 insensitive to the low amplitudes of variability seen clearly in HD 20884 and HD 20790. For example, guide star G2 is about 12.4 times fainter than HD 20884 so the Poisson noise is 3.5 times worse. Frequency analysis of the stars reveals that at frequencies above $4 \mathrm{~d}^{-1}$ (where there is no evidence of intrinsic variability in either star) the mean amplitude of the noise is about 4 times larger for G2 than for HD 20884, about as expected.

Figure presents binned light curves of HD 20884 and HD 20790, as well as the original unbinned photometry of HD 20884. The values of the point-to-point scatter of the $26.25 \mathrm{~s}$-exposure data for the two stars are $3.6 \mathrm{mmag}$ and $8.9 \mathrm{mmag}$, respectively. The point-to-point scatters of the binned data are $0.225 \mathrm{mmag}$ and $0.303 \mathrm{mmag}$. To illustrate the stability of the MOST Guide Star photometry for stars of brightness comparable to or greater than HD 20884 and HD 20790, we also plot in Figure a guide star, HD 146490 ( $V=7.2 \mathrm{mag}$ ), observed in the same fashion under similar spacecraft and orbital conditions during a MOST Fabry Imaging run on the star $\epsilon$ Oph. The HD 146490 photometry has been binned in the same way; the point-topoint scatter is $0.122 \mathrm{mmag}$. The $\gamma$ Dor variability of HD 20790 will be reported in detail in a separate paper. We concentrate here on the variability of HD 20884.

The HD 20884 photometry used in this paper is available on the MOST Public Data Archive $^{2}$ through the Science link.

\section{Spectral classification of HD 20884 and the other guide stars}

Like many of the relatively "anonymous" MOST guide stars, there is very little detailed information in the literature on HD 20884, beyond a Simbad spectral classification of $\mathrm{K} 0$ and a Hipparcos parallax of $7.18 \pm 0.97$ mas.

Due to the evidence for intrinsic variability in HD 20884 and at least one of the other guide stars, it was important to verify or refine their spectral types, and obtain luminosity classes. In November 2004, spectra were obtained by one of us, SMR, using the Cassegrain spectrograph on the 1.88-m telescope of the David Dunlap Observatory. The spectral resolving power of the data is $\sim 9000$ and the $S / N$ approximately 60 . The spectra of HD 20884 and HD 20790 are shown in Figure 2, classified as a K2 giant and F4 subgiant, respectively, with an estimated uncertainty of 2 sub-types. Previous classifications in the literature were $\mathrm{KO}$ and $\mathrm{F} 0$, with no luminosity classes. The classifications of all four guide stars are given in Table 1.

The F4IV classification of HD 20790 is consistent with our identification of this star as a $\gamma$ Doradus pulsator. The K2III classification of HD 20884 means the timescales and amplitudes of the observed photometric variability in this star are consistent with $p$-mode pulsations.

\footnotetext{
${ }^{2}$ http://www.astro.ubc.ca/MOST
} 


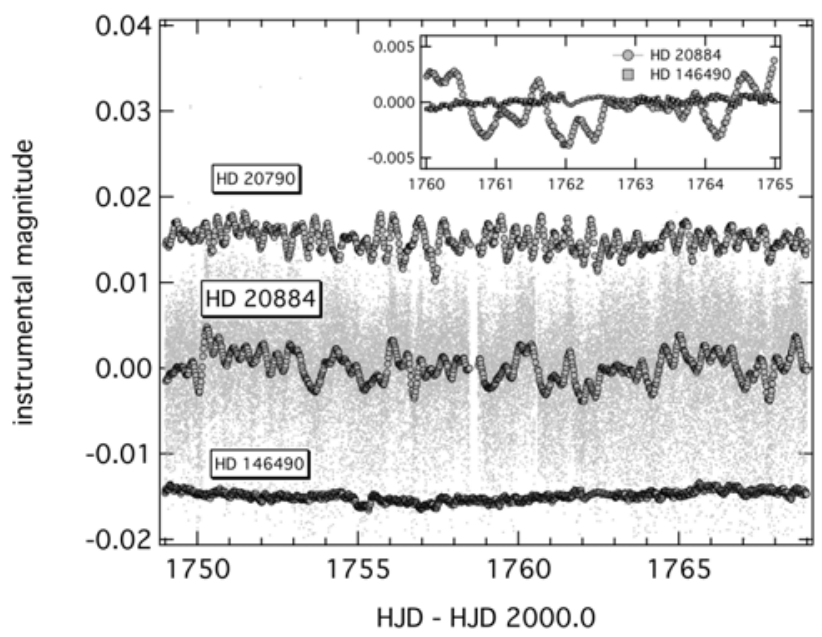

Figure 1: MOST photometry of the stars HD 20884 and HD 20790, compared to the constant guide star HD 146490 observed by MOST under similar conditions. Time is shifted for HD 146490 to overlay the other observations. The circles are averages of 50 consecutive 26.25-s exposures (covering about $22 \mathrm{~min}$ each); the unaveraged measurements of HD 20884 are shown as small grey dots The inset is an enlargement of 5 days of data for HD 20884 and HD 146490.

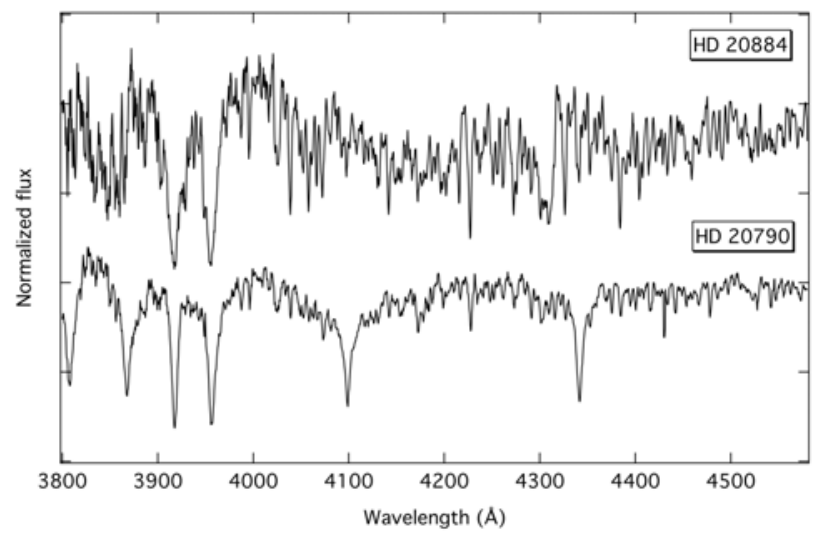

Figure 2: Classification spectra of HD 20884 and HD 20790 (G1) obtained with the $1.88 \mathrm{~m}$ telescope at DDO. The spectra were classified as K2 giant (HD 20884) and F4 sub-giant, with estimated uncertainties of 2 sub-types (G1). 


\section{Frequency analysis of HD 20884}

Before the frequency analysis, to identify any obvious outliers, the data were subjected to a $3 \sigma$ clipping. Less than $0.6 \%$ of the points were rejected in all four guide star time series. The low-frequency ranges of the Fourier amplitude spectra for HD 20884 and HD 20790 are plotted with the same scale in Figure 3, along with the spectrum of the bright constant guide star HD 146490. The spectral window, shown in the upper right corner, demonstrates that there is no aliasing thanks to the nearly continuous time coverage of the MOST data ( $96 \%$ duty cycle).

Guide star HD 20790 cannot be used as a direct photometric comparison due to its intrinsic variability and the other two guide stars cannot be used because of their faintness relative to HD 20884. However, they can help to identify any spurious frequencies due to orbital or instrumental artifacts that may be present in the Guide Star data.

The most dominant variations occur at the MOST satellite orbital frequency of about $14.2 \mathrm{~d}^{-1}(165 \mu \mathrm{Hz})$ and harmonics which are due to modulation of scattered Earthshine on the instrument focal plane. Fortunately, the clean spectral window of the data (see the inset in Figure 3) prevents from spilling sidelobes into the frequency range of interest for HD 20884. For the same reason, any low-level long-term instrumental drifts below $1 \mu \mathrm{Hz}$ have no sidelobes that might interfere with our analysis.

There is sometimes weak modulation of stray light in MOST data with frequencies of 1,2 and $3 \mathrm{~d}^{-1}$ since the satellite's Sun-synchronous orbit brings the sub-sattelite point to coincidence with nearly the same area on ground (and hence a similar reflective feature in the Earth's albedo) after 1 day. The $2 \mathrm{~d}^{-1}$ artifact is present in the three fainter guide stars and can be seen in the HD 20790 amplitude spectrum in Figure 3. The $1 d^{-1}$ feature is present in the G2 and G3 data, but not in HD 20790. The $3 \mathrm{~d}^{-1}$ artifact is present in the HD 20790 data and very weakly in G3. There are no significant peaks at any of the cycle/day stray light modulation frequencies in the HD 20884 photometry.

To identify significant frequencies in the guide star data sets, the routine SigSpec $^{3}$ (Reegen 2007) was used to detect periodic signals in data sets. It relies on an exact analytical solution for the probability that a given DFT (Discrete Fourier Transform; Deeming 1975) amplitude is generated by white noise. On average, an amplitude signal-to-noise ratio of 4 roughly corresponds to a SigSpec significance value of 5.46. This means that an amplitude of four times the noise level would appear by chance in one out of $10^{5.46}$ cases, assuming white noise.

SigSpec automatically identifies the frequency associated with maximum significance in the frequency range of interest, determines the amplitude and phase for this peak frequency using a least-squares fit, and subtracts the resulting sinusoid from the light curve (prewhitening). The residuals are used as input for the next significance calculation. The result of this step-by-step procedure is a list of significant signal components forming a multiperiodic fit to the time series.

The frequency analysis was performed from 0 to $15 \mathrm{~d}^{-1}$ down to a significance limit $s i g \geq 4.0$ (equivalent to a $\mathrm{s} / \mathrm{N}$ ratio of about 3.4 ). It identifies $53,22,15$,

\footnotetext{
${ }^{3}$ Significance Spectrum, http://www.astro.univie.ac.at/SigSpec
} 


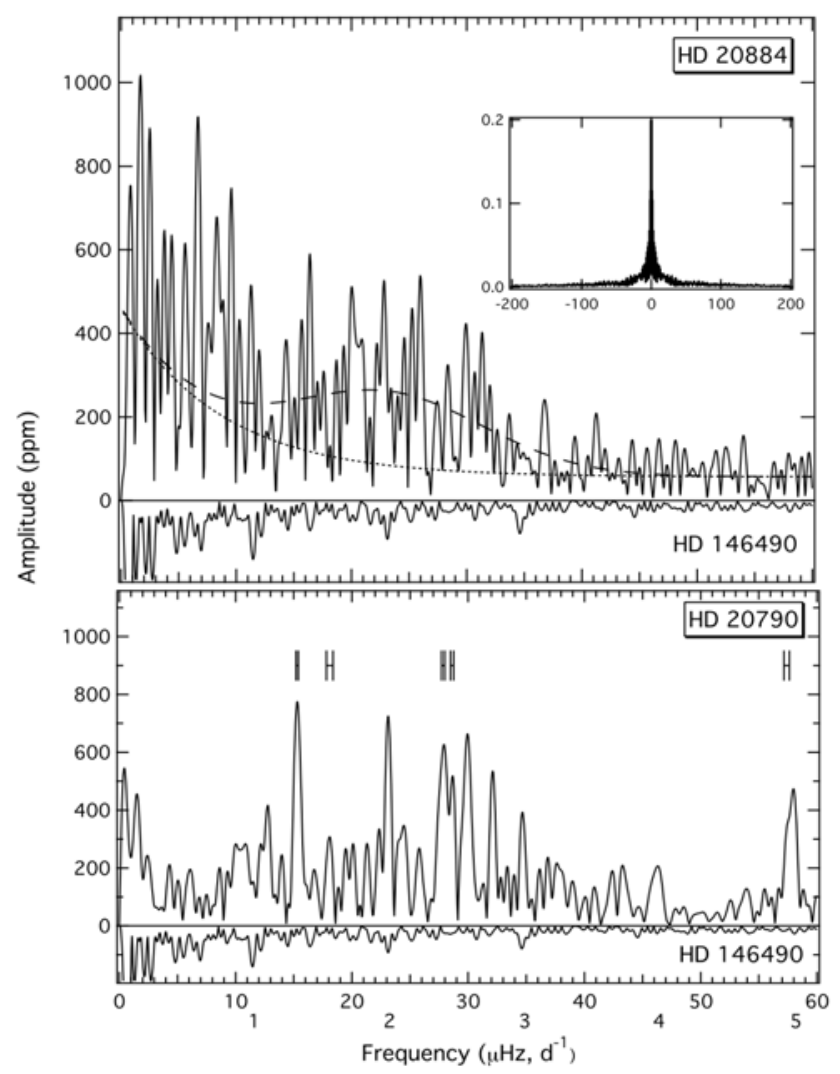

Figure 3: Fourier amplitude spectra of HD 20884 (K2III) and HD 20790 (F8), and that of a constant MOST guide star, HD 146490 (A2; inverted for easier comparison). All are shown at the same scale. The dashed line corresponds to a function (described in the text) fitted to the amplitude spectrum. The dotted line shows the exponential and white noise part of the fit. The spectral window is shown in the inset; note the truncated amplitude scale. For HD 20790 the identified frequencies and their uncertainty ranges (a function of the $\mathrm{S} / \mathrm{N}$ of each peak) are labelled by the connected vertical bars at the top of the panels.

and 12 formally significant peaks for HD 20884, HD 20790, G2 and G3, respectively. These include the MOST orbital frequency near $14.2 \mathrm{~d}^{-1}$ and the 1,2 , and $3 \mathrm{~d}^{-1}$ stray light modulation features discussed above. To be conservative, we have rejected all frequencies that occur (within the uncertainties) in at least two guide stars, even recognising the relatively large inherent scatter in the faint stars G2 and G3 and the clear intrinsic $\gamma$ Dor variability of HD 20790. (In the latter star, there are still 5 


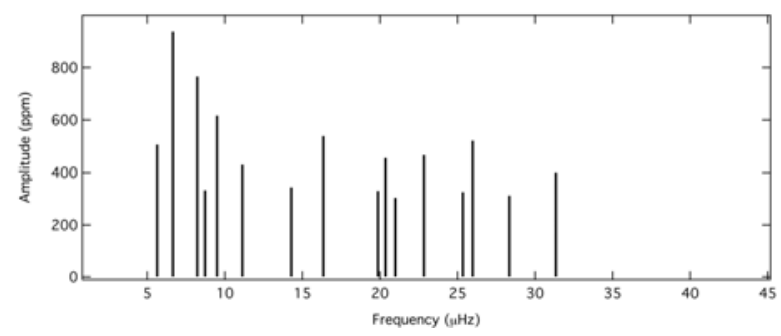

Figure 4: Schematic Fourier amplitude spectrum of the identified significant frequencies in the HD 20884 time series, after very conservative rejection of potential artifacts.

frequencies that survive this rigorous cut, and other almost certainly intrinsic peaks that happen to overlap with the dense frequency spectrum of HD 20884.) At the lowest frequencies, we are likely rejecting some intrinsic variability of HD 20884 that overlaps with genuine turbulent variability in the other stars including a giant and subgiant. The frequency uncertainties are estimated according to Kallinger et al. (2008a), who define the upper limit for the frequency error of a least-square sinusoidal fit to be $(T \cdot \sqrt{s i g})^{-1}$ where $T$ is the data set length. We ultimately reject 27 out of the 53 frequencies identified in HD 20884.

Figure 3 also illustrates the problem of safely extracting intrinsic frequencies in the low frequency domain. The amplitude spectrum seems to show some power excess from about $15-40 \mu \mathrm{Hz}$ with a superposed frequency dependent noise source. The average amplitude in the range below $\sim 40 \mu \mathrm{Hz}$ is significantly higher (about 273ppm) than above $\sim 40 \mu \mathrm{Hz}$ (about 58ppm) and it is not clear which contribution comes from possibly yet undetected instrumental variations, granulation noise, artifacts due to short mode lifetimes, or a combination of all this. Furthermore, SigSpec is designed to handle white noise only and would overestimate the peak significances for frequency ranges with significant colored noise, like is the case for a granulation signal. Because it is extremely difficult to fit a model to a set of frequencies that include even a small number of spurious peaks, we restrict our list of frequencies to those exceeding a significance value of 15 .

However, to test if our method of peak selection is consistent with a frequencydependent noise source, we need to estimate the contributions coming from white noise, a frequency-dependent signal due to granulation, and pulsation. We therefore assume the amplitude spectrum to be a superposition of white noise, an exponential decay, and a power excess hump approximated by a Gaussian function and fit the corresponding function

$$
A(f)=a_{1}+a_{2} \cdot e^{-\frac{f}{a_{3}}}+a_{4} \cdot e^{-\left(\frac{f-a_{5}}{4 a_{6}}\right)^{2}}
$$

(where $a_{1}$ to $a_{6}$ are the coefficients to be fitted) to the amplitude spectrum (dashed line in Figure 3). We then compare the peak amplitudes to a local noise model 
Table 2: Frequencies, amplitudes (A) and significances $(\mathrm{S})$ detected in the light curve of HD 20884 after conservative rejection of potential artifacts. S/N ratios are determined in reference to a power law fit to the residual amplitude spectrum. Frequency uncertainties are given in brackets. Also listed are the corresponding mode degree $l$ and radial order $n_{p}$ of the best-fitting model.

\begin{tabular}{ccccccc}
\hline \hline \multicolumn{2}{c}{ Frequency } & $\mathrm{A}$ & $\mathrm{S}$ & $\mathrm{S} / \mathrm{N}$ & $l$ & $n_{p}$ \\
$\mathrm{~d}^{-1}$ & $\mu \mathrm{Hz}$ & $\mathrm{ppm}$ & & & & \\
\hline $0.487(0.008)$ & $5.64(0.09)$ & 505 & 41.3 & 2.0 & 1 & 1 \\
$0.575(0.004)$ & $6.65(0.05)$ & 937 & 131.8 & 3.9 & 1 & 2 \\
$0.711(0.005)$ & $8.22(0.06)$ & 765 & 89.7 & 3.6 & 0 & 1 \\
$0.821(0.006)$ & $9.50(0.07)$ & 615 & 60.3 & 3.2 & 1 & 3 \\
$0.962(0.009)$ & $11.13(0.10)$ & 428 & 30.1 & 2.6 & 0 & 2 \\
$1.235(0.011)$ & $14.29(0.13)$ & 343 & 19.3 & 2.6 & 0 & 3 \\
$1.413(0.007)$ & $16.35(0.08)$ & 537 & 46.2 & 4.6 & 2 & 4 \\
$1.717(0.011)$ & $19.87(0.13)$ & 329 & 18.1 & 3.4 & 0 & 5 \\
$1.759(0.008)$ & $20.36(0.10)$ & 454 & 33.3 & 4.8 & & \\
$1.814(0.012)$ & $20.99(0.14)$ & 303 & 15.4 & 3.3 & 1 & 7 \\
$1.973(0.008)$ & $22.83(0.10)$ & 465 & 35.0 & 5.4 & 0 & 6 \\
$2.190(0.012)$ & $25.34(0.13)$ & 325 & 17.6 & 4.2 & 0 & 7 \\
$2.245(0.007)$ & $25.98(0.09)$ & 520 & 43.4 & 6.8 & & \\
$2.449(0.012)$ & $28.34(0.14)$ & 311 & 16.1 & 4.3 & 0 & 8 \\
$2.708(0.009)$ & $31.34(0.11)$ & 400 & 26.1 & 5.9 & 0 & 9 \\
\hline
\end{tabular}

(dotted line in Figure 3) derived from the exponential decay and white noise part of the function $\left(a_{1}+a_{2} \cdot e^{-f / a_{3}}\right)$. Only two frequencies from our list have a $\mathrm{S} / \mathrm{N}$ ratio lower than 2. Consequently, we rejected them from our list of candidate frequencies. Note, that it might appear too optimistic to consider peaks with very low $\mathrm{S} / \mathrm{N}$ ratios to be intrinsic. But we have to mention that our local noise model is just a rough estimate and that it is quite conventional to use low $\mathrm{S} / \mathrm{N}$ peaks in the analyses of solar-type oscillations (see e.g., Carrier \& Eggenberger 2006 or Bonanno et al. 2008).

In conclusion, we are left with 15 frequencies for a subsequent analysis. They are listed in Table 2 and shown in a schematic Fourier amplitude spectrum in Figure 4.

\section{Asteroseismic Analysis}

To understand the nature of the oscillations, we compare the observed frequencies with the eigenspectra determined from stellar models. We calculated radial and nonradial mode frequencies of a grid of standard stellar models distributed along evolutionary tracks on the giant branch of the HR-Diagram, constructed with the Yale Rotating Evolutionary Code (Guenther et al. 1992). The code uses the OPAL equation of state 

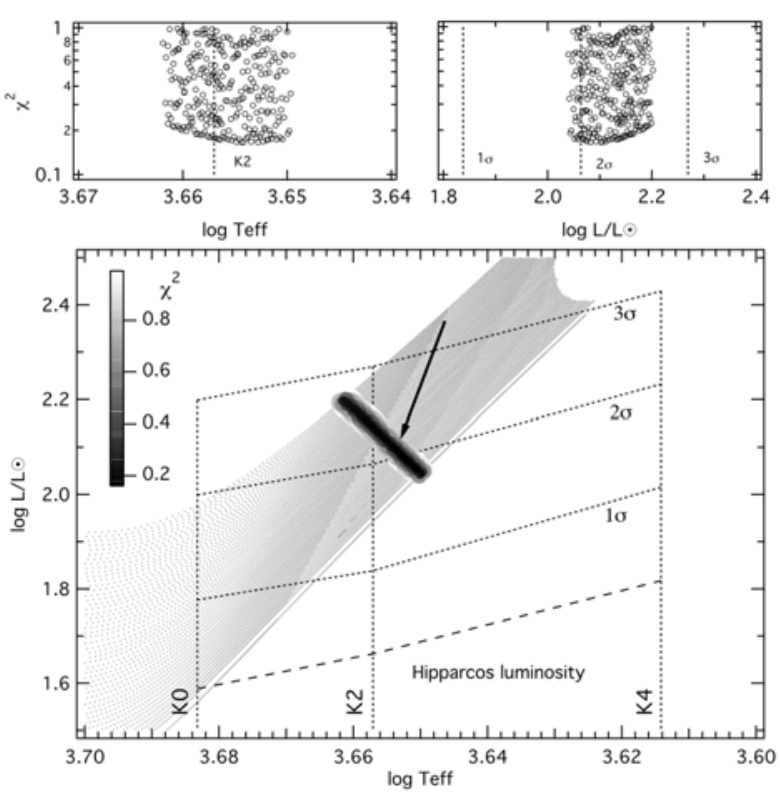

Figure 5: Theoretical HR-diagram showing the suspected location of HD 20884 and a subset of the stellar model grid used for the pulsation analysis (light grey dots). The grey scale gives the $\chi^{2}$ values from the differences between the observed and model frequencies, where the scale is limited to values less than 1.0. The position of the best-fitting model is indicated by an arrow. The top panels illustrate $\chi^{2}$ as a function of model temperature and luminosity, respectively.

(Iglesias \& Rogers 1996), OPAL opacities in the interior (Rogers \& Iglesias 1994), and the low-temperature opacities from Alexander \& Ferguson (1994) in the envelope. Diffusion of helium and heavy elements is not included. The model pulsation spectra were generated by the nonradial, nonadiabatic stellar pulsation program developed by Guenther (1994) using the Henyey relaxation method to solve the linearised oscillation equations.

Giant stars possess closely spaced $g$-modes at frequencies that overlap the frequency range of the $p$-modes. Consequently, there is strong mode bumping where the nonradial $p$-modes interfere with $g$-modes (see Guenther et al. 2000). Hence any nonradial $p$-mode seen on the surface has a $g$-mode character in the interior with high radial order $\left(n_{g}=\sim 20\right.$ to 100$)$. These are the same modes identified as strongly trapped unstable modes by Dziembowski et al. (2001). The densely packed fluctuations in the radial displacement eigenfunctions near the stellar core heavily strain the numerical calculation and can lead to numerical instabilities. To avoid this complication, we force the code to ignore the inner $10 \%$ (in radius) of the stellar models. We 
found that the mode eigenfunctions in the outer $90 \%$ of the model are practically the same if the inner part of the model is used or not. However, the disadvantage of this "trick" is that the code does not derive the correct radial order in the $g$-mode part of the eigenfunction.

We computed radial and nonradial mode frequencies with mode degree $l$ up to 3 for more than 16000 models of intermediate mass stars with masses ranging from 1.35 to $3.5 \mathrm{M}_{\odot}$ (in steps of $0.02 \mathrm{M}_{\odot}$ ). The mass range used here was determined from initial test runs using more extensive grids covering the mass range 0.8 to $5.0 \mathrm{M}_{\odot}$. The model sequences start at temperatures around $5000 \mathrm{~K}$ and continue up to the initiation of the He-core burning at the end of the red giant phase. The initial helium and metal mass fractions were set to $(Y, Z)=(0.26,0.02)$. The mixing length parameter, adjusting the temperature gradient in the convective regions, was set to $\alpha=1.60$.

Following Guenther et al. (2005), a $\chi^{2}$ algorithm searches for close matches between the observed oscillation spectrum and the model frequencies. The quality of the match is given by

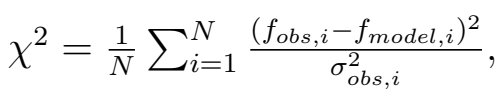

where $f_{o b s, i}$ is the observed frequency of the $i$ th mode and $f_{\text {model, } i}$ is the corresponding model eigenfrequency. $\sigma_{o b s, i}$ is the corresponding frequency uncertainty (see Table 2), and $\mathrm{N}$ is the total number of matched modes. $\chi^{2} \leq 1.0$ indicates that, on average, the model frequencies are within $1 \sigma$ of the uncertainty of the observed frequencies. Because we have no way of accurately estimating the frequency uncertainties or mode lifetimes, we have simply used the frequency uncertainties the signals would have if they are coherent stable sinusoids as listed in Table 2. Our $\chi^{2}$, therefore, in this context is only a relative measure of how well the models fit the observed frequencies. To aid in visualizing the best fitting models we have selected models with $\chi^{2} \leq 1$ to show those models whose frequencies lie within our assumed uncertainties of the model frequencies. We stress that without a proper and accurate estimate of mode lifetimes and uncertainties, we cannot provide a statistical measure of how well the models are constrained. For more details, see Guenther et al. (2005).

We started our fitting procedure by restricting the models to $l=0$ and found that it is not possible to find a model in our grid whose radial $p$-modes fit more than 8 of 15 observed frequencies. Only when also allowing to search for nonradial modes are we able to fit 13 of the 15 observed frequencies. In this case, our fitting procedure yields a ridge of models with $\chi^{2} \leq 1$ with a well defined large frequency separation of about $2.9 \mu \mathrm{Hz}$ (tilted grey-scaled bar in Figure 5). The formally best-fitting model (indicated by the arrow in Figure 5 ), with a $\chi^{2}=0.18$, has a mass of $2.84 \mathrm{M}_{\odot}$, an effective temperature of $4508 \mathrm{~K}$ and a luminosity of $126.5 \mathrm{~L} \odot$. The effective temperature of this model is compatible with a typical K2 giant star (Gray 1992). 
As can be seen in Figure 5, the best-fitting models lie above the luminosities based on the Hipparcos parallax. The absolute visual magnitude $M_{V}=1.31$ of HD 20884 can be derived from its average $V$ magnitude of $7.47 \mathrm{mag}$, the maximum-likelihood distance of $162.2 \pm 25.9 \mathrm{pc}$ (Hipparcos catalog) and the interstellar absorption $A_{V}$ of $0.11 \pm 0.03$ (Arenou et al. 1992). The bolometric correction $B C$ for a K2 giant star (4540 K; Gray 1992) is -0.715 (Reed 1998; Cayrel et al. 1997) resulting in an estimated luminosity of $45.9 L_{\odot}$ according to

$$
L / L_{\odot}=79.43 \times 10^{-0.4 \cdot\left(M_{V}+B C\right)} .
$$

A luminosity of about $45.9 \mathrm{~L}_{\odot}$ is very different from the luminosity of our best-fitting model. We note that there is, for a star of the given apparent magnitude, an unusual variety of published values for the $V$ magnitude of HD 20884 in the recent literature ranging from $7.02 \mathrm{mag}$ (NPM1 Catalog; Klemola et al. 1987) to $8.0 \mathrm{mag}$ (SAO Star Catalog). We speculate that this is intrinsic to the star due to, e.g., unknown binarity or surface spots. The bolometric correction for cool stars depends strongly on the temperature, and we estimate its uncertainty to be \pm 0.1 . The spread of published spectral classifications of HD 20884 corresponds to a temperature range of $4820 \mathrm{~K}$ $4110 \mathrm{~K}$ (Gray 1992). Our new spectral classification gives the temperature range we plot in Figure 5. Given these uncertainties, the expected luminosity of HD 20884 falls in a range from $38.7 L_{\odot}$ for a $\mathrm{K} 0$ giant to $65.6 L_{\odot}$ for a $\mathrm{K} 4$ giant.

The best-fitting model from our standard grid is more than $2 \sigma$ above the Hipparcosbased luminosity. We therefore investigated the influence of the mixing length parameter $\alpha$ and chemical composition on the eigenspectrum and found that it is indeed possible to find a significantly less luminous model that matches the observed frequencies. E.g., decreasing the mixing length to $\alpha=1.1$ results in an approximately $25 \%$ less luminous best-fitting model within $1 \sigma$ uncertainty of the Hipparcos-based luminosity and the cool temperature boundary in the HR-diagram, whereas the corresponding eigenspectra is practically the same. We have to mention that changing the model's chemical composition has a similar but less distinctive effect. Unfortunately temperature and luminosity of HD 20884 are poorly determined and no estimate for the metal abundance is given in the literature. Consequently, we are not able to reasonably narrow the parameter space for our model search to find a solution that fits the observed frequencies as well as other independently derived parameters (as it was done for $\epsilon$ Oph in Kallinger et al. 2008b).

The 15 frequencies listed in Table 2 are plotted in an echelle diagram in Figure 6 with a folding frequency of $2.9 \mu \mathrm{Hz}$. Note that the autocorrelation for the frequency range $15-40 \mu \mathrm{Hz}$ yields $3.0 \pm 0.2 \mu \mathrm{Hz}$, consistent with the large spacing of the fitted model. The filled black circles indicate the observed frequencies matched by the model, and the open circles the remaining frequencies. The error bars indicate the assumed frequency uncertainty for the individual modes, which we stress is introduced only to provide a point of reference for model comparisons. They do not account for mode lifetimes comparable to the observing run duration. The other symbols are the model eigenfrequencies. The mode identifications are given in Table 2. 


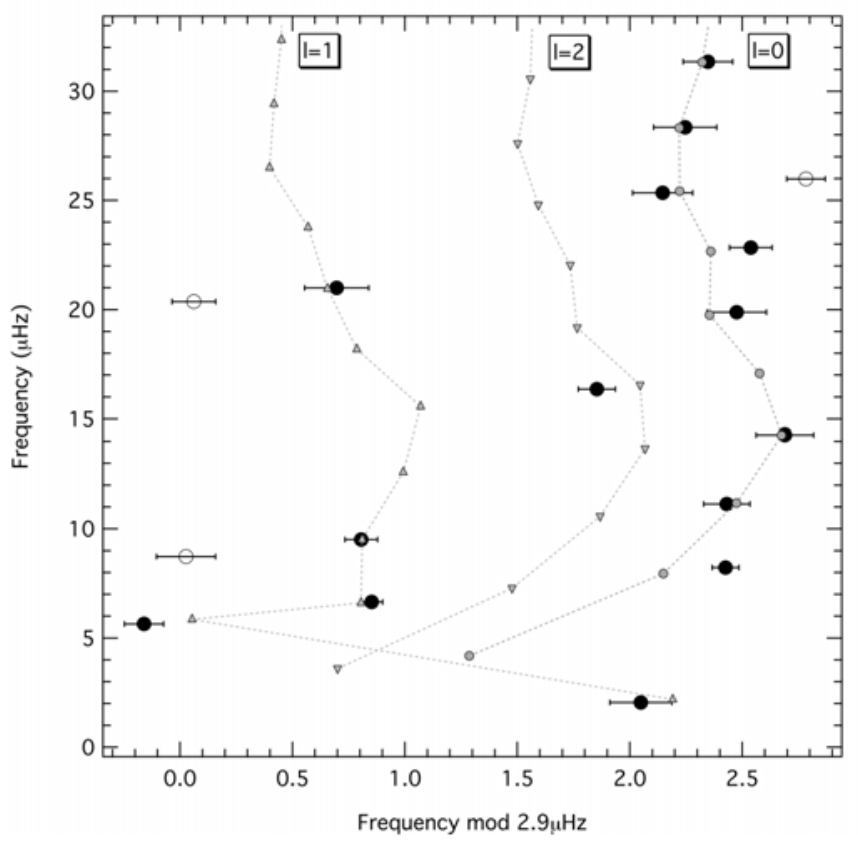

Figure 6: Echelle diagram of the 15 most significant observed oscillation frequencies used to constrain the fit and the best fitting model modes. The observed photometric modes are shown as circles with error bars indicating observational uncertainties. Filled circles indicate the 13 observed frequencies matched by model modes and open circles the remaining 2 frequencies. The model modes are given by grey symbols connected by line segments.

\section{Internal structure of HD 20884}

The seismic models of the K0 giant $\alpha$ UMa by Guenther et al. (2000) show that the structure of red giants in the expected temperature and luminosity range of HD 20884 is defined by a burned-out helium core, a hydrogen-burning shell, and a deep convective envelope. Figure 7 illustrates the variation of the squared Lamb frequency $L^{2}$ and the squared Brunt-Väisälä frequency $N^{2}$ along the radius of the best-fitting model. Whereas the Lamb frequency corresponds to the minimum frequency at which an acoustic wave can propagate in a given medium, the Brunt-Väisälä frequency is the frequency at which a liquid bubble would wobble, driven by its natural buoyancy, if slightly displaced from its equilibrium. The propagation diagram in Figure 7 shows in which regions of the stellar interior $p$-modes or $g$-modes can propagate. $g$-modes can only exist in regions where their frequency is below the Lamb frequency and the Brunt-Väisälä frequency, whereas $p$-modes can propagate in regions where their 


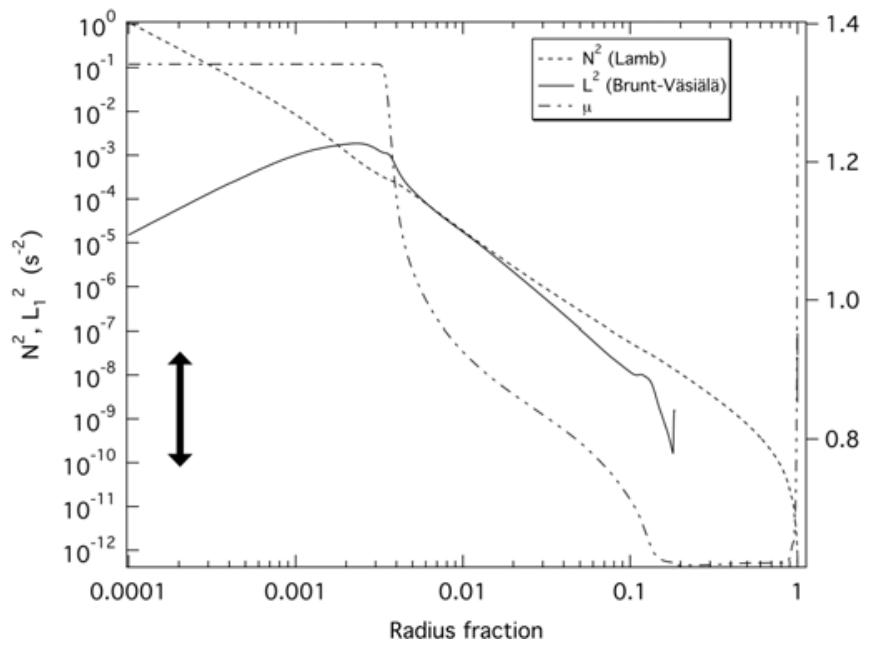

Figure 7: Squared Lamb and squared Brunt-Väisälä frequency along the radius for the best-fitting model. The squared Brunt-Väisälä frequency is negative in the convective envelope and hence not plotted on a logarithmic scale in the range of the convective envelope. The arrow indicates the frequency range of the observations. Also plotted is the mean molecular weight $\mu$.

frequency is above the Lamb frequency and the Brunt-Väisälä frequency. Contrary to normal main sequence stars, where the $p$-mode frequency range is separated from the $g$-mode range, the internal structure of HD 20884 allows the existence of $g$-modes at frequencies that overlap the $p$-mode frequency domain. It turns out that all observed nonradial $l=1$ and 2 modes (the frequency range of the observed modes is indicated by an arrow in Figure 7) are high-order $g$-modes with a $p$-mode character in the outer layers, so-called mixed modes.

\section{Mode lifetimes}

Contrary to $p$-modes in the classical instability strip, which are driven by the $\kappa$ mechanism and which can coherently oscillate for millions of years, damped and stochastically excited modes, present in the Sun and apparently in solar-type stars and cool giants, have much shorter lifetimes. For the Sun, the measured lifetimes are on the order of a few days. The Fourier transform (which is defined for a coherent signal) of an unstable signal with changing amplitudes and/or phases, like a damped and re-excited mode, produces artificial beat frequencies. Consequently, the line profile in the amplitude spectrum becomes Lorentzian. If the damping time is comparable to the data set length, only individual and randomly separated side lobes are produced by the damped and randomly re-excited modes. In this case, it is not possible to distinguish signals produced by short-lived modes from those of closely spaced long-lived modes. 
The few previously available sets of observations of red giant $p$-mode oscillations may still not be adequate to test the predictions of strong damping of nonradial modes made by Dziembowski et al. (2001) and to reinforce their claim that radial modes dominate the frequency spectrum. Also the question of the length of $p$-mode lifetimes in cool giants is not yet answered. For example, Stello et al. (2006) claim mode lifetimes of about 2 days for the $\mathrm{G} 7$ giant $\xi$ Hydrae, in contradiction to Kallinger et al. (2008b) who found indications that the mode lifetimes could be as long as 10 to 20 days in the G9.5 giant $\epsilon$ Oph which is consistent with theoretically predicted lifetimes of 15 to 20 days for late $G$ type giants (Houdek et al. 2002).

However, we follow the approach of Kallinger et al. (2008b) and assume the detected frequencies as individual radial and nonradial modes whose lifetimes are comparable to the length of the observations. Kjeldsen et al. (2005) have shown that, for solar-type oscillations, the scatter of the observed frequencies about the true mode frequencies is a function of the mode lifetime and the signal-to-noise ratio of the observed signal. Kallinger et al. (2008b) picked up this idea and discovered that the observed frequency scatter also correlates with the square root of the duration of the observations. From their Figure 6 we derive an average mode lifetime of about 10 days for our HD 20884 observations but have to mention that this is just a rough estimate.

\section{Conclusions}

For the K giant HD 20884, we have discovered in MOST photometry a rich oscillation spectrum in a frequency and amplitude range where pulsations have already been reported for similar giants. Of the 15 identified frequencies, 13 are compatible with radial $p$-modes and nonradial mixed modes. Using only asteroseismic techniques, we estimate the effective temperature and find it to be compatible with the spectral classification we have made based on DDO spectra. If our analysis is correct, the mode lifetimes are most likely longer than 10 days, consistent with the theoretical predictions made by Houdek et al. (2002) for a star like HD 20884.

However, our best-fitting model is more than twice as luminous than one would expect from Hipparcos parallax. But we have shown that when changing the mixing length to $\alpha=1.1$, models that fit the observed modes are under-luminous by more than $1 \sigma$. The mixing length is a single parameter that adjusts the temperature adiabat of the convective envelope. Somewhat similar changes can be brought about by altering the opacities or the composition, or using an improved model of convection. In other words, the low temperature opacities, the composition, and the mixing length parameter used here to fix the structure of the surface layers, are probably not unique. But with more accurate observations, we should be able to determine specifically what aspects of the models of giant stars need to be improved.

Acknowledgments. T.K., M.H.,P.R. and W.W.W. are supported by the Austrian Fonds zur Förderung der wissenschaftlichen Forschung (FWF) within the project The Core of the HR diagram (P17580-N02), and the Bundesministerium für Verkehr, 
Innovation und Technologie (BM.VIT) via the Austrian Agentur für Luft- und Raumfahrt (FFG-ALR). The Natural Science and Engineering Research Council of Canada supports the research of D.B.G, J.M.M., A.F.J.M., S.M.R., and G.A.H.W.; A.F.J.M. is also supported by FQRNT (Québec). R.K. is supported by the Canadian Space Agency. D.S. is supported by the National Science Foundation.

\section{References}

Aerts, C., De Cat, P., Kuschnig, R., et al. 2006, ApJ 642, 165

Alexander, D. R., \& Ferguson, J. W. 1994, ApJ 437, 879

Arenou, F., Grenon, M., \& Gómez, A. 1992, A\&A 258, 104

Barban, C., Matthews, J. M., De Ridder, J., et al. 2007, A\&A 468, 1033B

Bouchy, F., \& Carrier, F. 2003, Ap\&SS 284, 21

Bonanno, A., Benatti, S., Claudi, R., et al. 2008, 2008arXiv0801.4446B

Carrier, F., \& Eggenberger, P. 2006, A\&A 450, 695

Cayrel, R., Castelli, F., Katz, D., et al. 1997, ESA SP-402, 433

Deeming, T. J. 1975, Ap\&SS 36, 137

Dziembowski, W. A., Gough, D. O., Houdek, G. 2001, MNRAS 328, 601

Frandsen, S., Carrier, F., Aerts, C., et al. 2002, A\&A 394, L5

Gray, D. F. 1992, The observation and analysis of stellar photospheres (Cambridge: Cambridge University Press)

Guenther, D. B., Demarque, P., Kim, Y. -C., \& Pinsonneault, M. H. 1992, ApJ 387, 372

Guenther, D. B. 1994, ApJ 422, 400

Guenther, D. B., Demarque, P., Buzasi, D., et al. 2000, ApJ 530, L45

Guenther, D. B., Kallinger, T., Reegen, P., et al. 2005, ApJ 635, 547

Handler, G. 2005, JApA 26, 24

Hekker, S., Aerts, C., De Ridder, J., \& Carrier, F. 2006, A\&A 458, 931

Houdek, G., \& Gough, D. O. 2002, MNRAS 336, L65

Iglesias, C. A., \& Rogers, F. J. 1996, ApJ 464, 943

Kallinger, T., Reegen, P., \& Weiss, W. W. 2008a, arXiv0801.0683K

Kallinger, T., Guenther, D. B., Matthews, J. M., et al. 2008b, A\&A 478, 497

Kallinger, T., Zwintz, K., Pamyatnykh, A. A., et al. 2005, A\&A 433, 267

Kjeldsen, H., Bedding, T. R., Butler, R. P., et al. 2005, ApJ 635, 1281

Klemola, A. R., Hanson, R. B., \& Jones, B. F. 1987, AJ 94, 501

Matthews, J. M., Kuschnig, R., Guenther, D. B., et al. 2004, Nature 430, 51

Reed, C. 1998, JRASC 669, 36

Reegen, P., Kallinger, T., Frast, D., et al. 2006, MNRAS 367, 1417 
Reegen, P. 2007, A\&A 467, 1353

De Ridder, J., Barban, C., Carrier, F., et al. 2006, A\&A 448, 689

Rogers, F. J., \& Iglesias, C. A. 1994, Science 263, 50

Rowe, J. F., Matthews, J. M., Kuschnig, R., et al. 2006, MmSAI 77, 282

Rucinski, S. M., Walker, G. A. H., Matthews, J. M., et al. 2004, PASP 116, 1093

Stello, D., Kjeldsen, H., Bedding, T. R., \& Buzasi, D. 2006, A\&A 448, 709

Saio, H., Kuschnig, R., Gautschy, A., et al. 2006, ApJ 650, 1111

Walker, G. A. H., Matthews, J. M., Kuschnig, R., et al. 2003, PASP 115, 1023

Walker, G. A. H., Kuschnig, R., Matthews, J. M., et al. 2005, ApJ 635, 77

Walker, G. A. H, Croll, B., Kuschnig, R., et al. 2007, ApJ 659, 1611 
